\title{
Recovering Affine Deformations of Fuzzy Shapes
}

\author{
Attila Tanács ${ }^{1, \star}$, Csaba Domokos ${ }^{1}$, Nataša Sladoje ${ }^{2, \star \star}$, Joakim Lindblad $^{3}$, \\ and Zoltan Kato ${ }^{1}$ \\ 1 Department of Image Processing and Computer Graphics, \\ University of Szeged, Hungary \\ \{tanacs, dcs, kato\}@inf.u-szeged.hu \\ 2 Faculty of Engineering, University of Novi Sad, Serbia \\ sladoje@uns.ns.ac.yu \\ 3 Centre for Image Analysis, Swedish University of Agricultural Sciences, \\ Uppsala, Sweden \\ joakim@cb.uu.se
}

\begin{abstract}
Fuzzy sets and fuzzy techniques are attracting increasing attention nowadays in the field of image processing and analysis. It has been shown that the information preserved by using fuzzy representation based on area coverage may be successfully utilized to improve precision and accuracy of several shape descriptors; geometric moments of a shape are among them. We propose to extend an existing binary shape matching method to take advantage of fuzzy object representation. The result of a synthetic test show that fuzzy representation yields smaller registration errors in average. A segmentation method is also presented to generate fuzzy segmentations of real images. The applicability of the proposed methods is demonstrated on real X-ray images of hip replacement implants.
\end{abstract}

\section{Introduction}

Image registration is one of the main tasks of image processing, its goal is to find the geometric correspondence between images. Many approaches have been proposed for a wide range of problems in the past decades [1]. Shape matching is an important task of registration. Matching in this case consists of two steps: First, an arbitrary segmentation step provides the shapes and then the shapes are registered. This solution is especially viable when the image intensities undergo strong nonlinear deformations that are hard to model, e.g. in case of X-ray imaging. If there are clearly defined regions in the images (e.g. bones or implants in X-ray images), a rather straightforward segmentation method can be used to define its shape adequately. Domokos et al. proposed an extension [2] to the

\footnotetext{
* Authors from University of Szeged are supported by the Hungarian Scientific Research Fund (OTKA) Grant No. K75637.

** Author is financially supported by the Ministry of Science of the Republic of Serbia through the Projects ON144029 and ON144018 of the Mathematical Institute of the Serbian Academy of Science and Arts.
}

A.-B. Salberg, J.Y. Hardeberg, and R. Jenssen (Eds.): SCIA 2009, LNCS 5575, pp. 735 744, 2009.

(C) Springer-Verlag Berlin Heidelberg 2009 
parametric estimation method of Francos et al. [3] to deal with affine matching of crisp shapes. These parametric estimation methods have the advantage of providing accurate and computationally simple solution, avoiding both the correspondence problem as well as the need for optimization.

In this paper we extend this approach by investigating the case when the segmentation method is capable of producing fuzzy object descriptions instead of a binary result. Nowadays, image processing and analysis methods based on fuzzy sets and fuzzy techniques are attracting increasing attention. Fuzzy sets provide a flexible and useful representation for image objects. Preserving fuzziness in the image segmentation, and thereby postponing decisions related to crisp object definitions has many benefits, such as reduced sensitivity to noise, improved robustness and increased precision of feature measures.

It has been shown that the information preserved by using fuzzy representation based on area coverage may be successfully utilized to improve precision and accuracy of several shape descriptors; geometric moments of a shape are among them. In [4] it is proved that fuzzy shape representation provides significantly higher accuracy of geometric moment estimates, compared to binary Gauss digitization at the same spatial image resolution. Precise moment estimation is essential for a successful application of the object registration method presented in 2] and the advantage of fuzzy shape representations is successfully exploited in the study presented in this paper.

In Section 2 we present the outline of the previous binary registration method [2] and extend it to accommodate fuzzy object descriptions. A segmentation method producing fuzzy object boundaries is described as well. Section 3 contains experimental results obtained during the evaluation of the method. In a study of 2000 pairs of synthetic images we observe the effect of the number of quantization levels of the fuzzy membership function to the precision of image registration and we compare the results with the binary case. Finally, we apply the registration method on real X-ray images, where we segmented objects of interest by an appropriate fuzzy segmentation method. This shows the successful adjustment of the developed method to real medical image registration tasks.

\section{Parametric Estimation of Affine Deformations}

In this section, we first review a previously developed binary shape registration method in the continuous space [2]. Since digital images are discrete, an approximative formula by discretization of the space is derived. The main contribution of this paper is in using a fuzzy approach when performing discretization. Instead of sampling the continuous image function at uniform grid positions, and performing binary Gauss discretization, we propose to perform area coverage discretization, providing a fuzzy object representation. We also describe a segmentation method that supports our suggested approach and produces objects with fuzzy boundaries. 


\subsection{Basic Solution}

Herein we briefly overview the affine registration approach from [2]. Let us denote the points of the template and the observation by $\mathbf{x}, \mathbf{y} \in \mathbb{P}^{2}$, respectively in the projective space. The projective space allows simple notation for affine transforms and assumes using of homogeneous coordinates. Since affine transformations never alter the third (homogeneous) coordinate of a point, which is therefore always equal to 1 , we, for simplicity, and without loss of generality, liberally interchange between projective and Euclidean plane, keeping the simplest notation.

Let $\mathbf{A}$ denote the unknown affine transformation that we want to recover. We can define the identity relation as follows

$$
\mathbf{A x}=\mathbf{y} \quad \Leftrightarrow \quad \mathbf{x}=\mathbf{A}^{-1} \mathbf{y} .
$$

The above equations still hold when a properly chosen function $\omega: \mathbb{P}^{2} \rightarrow \mathbb{P}^{2}$ is acting on both sides of the equations [2]:

$$
\omega(\mathbf{A x})=\omega(\mathbf{y}) \quad \Leftrightarrow \quad \omega(\mathbf{x})=\omega\left(\mathbf{A}^{-1} \mathbf{y}\right) .
$$

Binary images do not contain radiometric information, therefore they can be represented by their characteristic function $\mathbb{1}: \mathbb{P}^{2} \rightarrow\{0,1\}$, where 0 and 1 are assigned to the elements of the background and foreground respectively. Let $\mathbb{1}_{t}$ and $\mathbb{1}_{o}$ denote the characteristic function of the template and observation. In order to avoid the need for point correspondences, we integrate over the foreground domains $\mathcal{F}_{t}=\left\{\mathbf{x} \in \mathbb{P}^{2} \mid \mathbb{1}_{t}(\mathbf{x})=1\right)$ and $\mathcal{F}_{o}=\left\{\mathbf{y} \in \mathbb{P}^{2} \mid \mathbb{1}_{o}(\mathbf{y})=1\right)$ of the template and the observation, respectively, yielding [2]

$$
|\mathbf{A}| \int_{\mathcal{F}_{t}} \omega(\mathbf{x}) d \mathbf{x}=\int_{\mathcal{F}_{o}} \omega\left(\mathbf{A}^{-1} \mathbf{y}\right) d \mathbf{y} .
$$

The Jacobian of the transformation $(|\mathbf{A}|)$ can be easily evaluated as

$$
|\mathbf{A}|=\frac{\int_{\mathcal{F}_{o}} d \mathbf{y}}{\int_{\mathcal{F}_{t}} d \mathbf{x}} .
$$

The basic idea of the proposed approach is to generate sufficiently many linearly independent equations by making use of the relations in Eq. (11)-(2). Since A depends on 6 unknown elements, we need at least 6 equations. We cannot have a linear system because $\omega$ is acting on the unknowns. The next best choice is a system of polynomial equations. In order to obtain a system of polynomial equations from Eq. (2), the applied $\omega$ functions should be carefully selected. It was also shown in [2] that by setting $\omega(\mathbf{x})=\left(x_{1}^{n}, x_{2}^{n}, 1\right)$ Eq. (2) becomes

$$
|\mathbf{A}| \int_{\mathcal{F}_{t}} x_{k}^{n} d \mathbf{x}=\sum_{i=0}^{n}\left(\begin{array}{l}
n \\
i
\end{array}\right) \sum_{j=0}^{i}\left(\begin{array}{l}
i \\
j
\end{array}\right) q_{k 1}^{n-i} q_{k 2}^{i-j} q_{k 3}^{j} \int_{\mathcal{F}_{o}} y_{1}^{n-i} y_{2}^{i-j} d \mathbf{y},
$$

where $k=1,2 ; n=1,2,3$ and $q_{k i}$ denote the unknown elements of the inverse transformation $\mathbf{A}^{-1}$. 


\section{$2.2 \quad$ Fuzzy Object Representation}

The polynomial system of equations in Eq. (3) is derived in the continuous space. However, digital image space provides only limited precision for these derivations and the integral can only be approximated by a discrete sum over the pixels. There are many approaches for discretization of a continuous function. The easiest way to form a discrete image is by sampling the continuous function at uniform grid positions. This approach, leading to a binary image, is also known as Gauss centre point digitization, and is used in the previous study [2. An alternative way is to perform a fuzzy discretization of the image.

A discrete fuzzy subset $F$ of a reference set $X \subset \mathbb{Z}^{2}$ is a set of ordered pairs $F=\left\{\left((i, j), \mu_{F}(i, j)\right) \mid(i, j) \in X\right\}$, where $\mu_{F}: X \rightarrow[0,1]$ is the membership function of $F$ in $X$. The fuzzy membership function may be defined in various ways; its values reflect the levels of belongingness of pixels to the object. One useful way to define the membership function on a reference set in case when it is an image plane, is to assign a value to each image element (pixel) that is proportional to its coverage by the imaged object. In that way, partial memberships (values strictly between 0 and 1 ) are assigned to the pixels on the boundary of the discrete object.

Note that in the coefficients of the system of equations in Eq. (3) are the first, second and third order geometric moments of the template and observation. In general, moments of order $i+j$ of a continuous shape $\mathcal{F}=\left\{\mathbf{x} \in \mathbb{P}^{2} \mid \mathbb{1}(\mathbf{x})=1\right\}$ are defined as

$$
m_{i, j}(\mathcal{F})=\int_{\mathcal{F}} x_{1}^{i} x_{2}^{j} d \mathbf{x} .
$$

In the discrete formulation the geometric moments of order $i+j$ of a discrete fuzzy set $F$ can be used, defined as

$$
\tilde{m}_{i, j}(F)=\sum_{\boldsymbol{p} \in X} \mu_{F}(\boldsymbol{p}) p_{1}^{i} p_{2}^{j} .
$$

This equation can be used to estimate geometric moments of a continuous 2D shape. Asymptotic error bounds for moments of order up to 2, derived in [4], show that moment estimates calculated from a fuzzy object representation provide a considerable increase of precision as compared to estimates computed from a crisp representation, at the same spatial resolution.

If $F$ is fuzzy representation of $\mathcal{F}$, it follows that $m_{i, j}(\mathcal{F}) \approx \tilde{m}_{i, j}(F)$. Thus, by using Eq. (4)-(5) the integrals in Eq. (3) can be approximated as

$$
\begin{gathered}
\int_{\mathcal{F}_{t}} x_{k}^{n} d \mathbf{x} \approx \sum_{\boldsymbol{p} \in X_{t}} \mu_{F_{t}}(\boldsymbol{p}) p_{k}^{n} \text { and } \\
\int_{\mathcal{F}_{o}} y_{1}^{n-i} y_{2}^{i-j} d \mathbf{y} \approx \sum_{\boldsymbol{p} \in X_{o}} \mu_{F_{o}}(\boldsymbol{p}) p_{1}^{n-i} p_{2}^{i-j},
\end{gathered}
$$

and the Jacobian can be approximated as

$$
|\mathbf{A}|=\frac{m_{00}\left(\mathcal{F}_{o}\right)}{m_{00}\left(\mathcal{F}_{t}\right)} \approx \frac{\tilde{m}_{00}\left(F_{o}\right)}{\tilde{m}_{00}\left(F_{t}\right)}=\frac{\sum_{\boldsymbol{p} \in X_{o}} \mu_{F_{o}}(\boldsymbol{p})}{\sum_{\boldsymbol{p} \in X_{t}} \mu_{F_{t}}(\boldsymbol{p})} .
$$


$X_{t}$ and $X_{o}$ are the reference sets (discrete domains) of the (fuzzy) template and (fuzzy) observation image, respectively.

The approximating discrete system of polynomial equations can now be produced by inserting these approximations into Eq. (3):

$$
|\mathbf{A}| \sum_{\boldsymbol{p} \in X_{t}} \mu_{F_{t}}(\boldsymbol{p}) p_{k}^{n}=\sum_{i=0}^{n}\left(\begin{array}{c}
n \\
i
\end{array}\right) \sum_{j=0}^{i}\left(\begin{array}{l}
i \\
j
\end{array}\right) q_{k 1}^{n-i} q_{k 2}^{i-j} q_{k 3}^{j} \sum_{\boldsymbol{p} \in X_{o}} \mu_{F_{o}}(\boldsymbol{p}) p_{1}^{n-i} p_{2}^{i-j} .
$$

Clearly, the spatial resolution of the images affects the precision of this approximation. However, sufficient spatial resolution may be unavailable in real applications or, as it is expected in case of 3D applications, may lead to too large amounts of data to be successfully processed. On the other hand, it was shown in 4 that increasing the number of grey levels representing pixel coverage by a factor $n^{2}$ provides asymptotically the same increase in precision as an $n$ times increase of spatial resolution. Therefore the suggested approach, utilizing increased membership resolution, is a very powerful way to compensate for insufficient spatial resolution, while still preserving desired precision of moments estimates.

\subsection{Segmentation Method Providing Fuzzy Boundaries}

Application of the moment estimation method presented in [4] assumes a discrete representation of a shape such that pixels are assigned their corresponding pixel coverage values. Definition of such digitization is given in [5]:

Definition 1. For a given continuous object $\mathcal{F} \subset \mathbb{R}^{2}$, inscribed into an integer grid with pixels $p_{(i, j)}$, the $n$-level quantized pixel coverage digitization of $\mathcal{F}$ is

$$
\mathcal{D}_{n}(\mathcal{F})=\left\{\left((i, j), \frac{1}{n}\left\lfloor n \frac{A\left(p_{(i, j)} \cap \mathcal{F}\right)}{A\left(p_{(i, j)}\right)}+\frac{1}{2}\right\rfloor\right) \mid(i, j) \in \mathbb{Z}^{2}\right\},
$$

where $\lfloor x\rfloor$ denotes the largest integer not greater than $x$, and $A(X)$ denotes the area of a set $X$.

Even though many fuzzy segmentation methods exist in the literature, very few of them result in pixel coverage based object representations. With an intention to show the applicability of the approach, but to not focus on designing a completely new fuzzy segmentation method, we derive pixel coverage values from an Active Contour segmentation 6. Active Contour segmentation provides a crisp parametric representation of the object contour from which it is fairly straightforward to compute pixel coverage values. Such a straightforward derivation is not always possible, if other segmentation methods are used. The main point argued for in this paper is of a general character, and does not rely on any particular choice of segmentation method.

We have modified the SnakeD plugin for ImageJ by Thomas Boudier [7] to compute pixel coverage values. The snake segmentation is semi-automatic, and requires that an approximate starting region is drawn by the operator. Once the 
snake has reached a steady state solution, the snake representation is rasterized. Each pixel close to the snake boundary is given partial membership to the object proportional to how large part of that pixel is covered by the segmented object. The actual computation is facilitated by a $16 \times 16$ supersampling of the pixels close to the object edge and the pixel coverage is approximated by the fraction of sub-pixels that fall inside the object.

\section{Experimental Results}

When working with digital images, we are limited to a finite number of levels to represent fuzzy membership values. Using a database of synthetic binary shapes, we examine the effect of the number of quantization levels to the precision of registration and compare them to the binary case. The pairs of corresponding synthetic fuzzy shapes are obtained by applying known affine transformations. Therefore the presented registration results for synthetic images are neither dependent nor affected by a segmentation method. Finally, the proposed registration method is tested on real X-ray images, incorporating the fuzzy segmentation step.

\subsection{Quantitative Evaluation on Synthetic Images}

The performance of the proposed algorithm has been tested and evaluated on a database of synthetic images. The dataset consists of 39 different shapes and their transformed versions, a total of 2000 images. The width and height of the images were typically between 500 and 1000 pixels. The transformation parameters were randomly selected from uniform distributions. The rotation parameter was not restricted, any value was possible from $[0,2 \pi)$. Scale parameters varied between $[0.5,1.5]$, shear parameters between $[-1,1]$. The maximal translation value was set to 150 pixels. The templates were binary images, i.e. having either 0 or 1 fuzzy membership values. The fuzzy border representations of the observation images were generated by using $16 \times 16$ supersampling of the pixels close to the object edge and the pixel coverage was approximated by the fraction of subpixels that fall inside the object. The fuzzy membership values of the images were quantized and represented by integer values having $k$-bit $(k=1, \ldots, 8)$ representation. Some typical examples of these images and their registration accuracies are shown in Fig. 1.

In order to quantitatively evaluate the results, we have defined two error measures. The first error measure (denoted by $\epsilon$ ) is the average distance in pixels between the true $(\mathbf{A p})$, and recovered $(\widehat{\mathbf{A}} \mathbf{p})$ positions of the transformed pixels over the template. This measure is used for evaluation on synthetic images, where the true transformation is known. Another measure is the absolute difference (denoted by $\delta$ ) between the registered template image and the observation image.

$$
\epsilon=\frac{1}{m} \sum_{\mathbf{p} \in T}\|(\mathbf{A}-\widehat{\mathbf{A}}) \mathbf{p}\|, \quad \text { and } \quad \delta=\frac{|R \triangle O|}{|R|+|O|},
$$

where $m$ is the number of template pixels, $\triangle$ means the symmetric difference, while $R$ and $O$ denote the set of pixels of the registered shape and the observation 

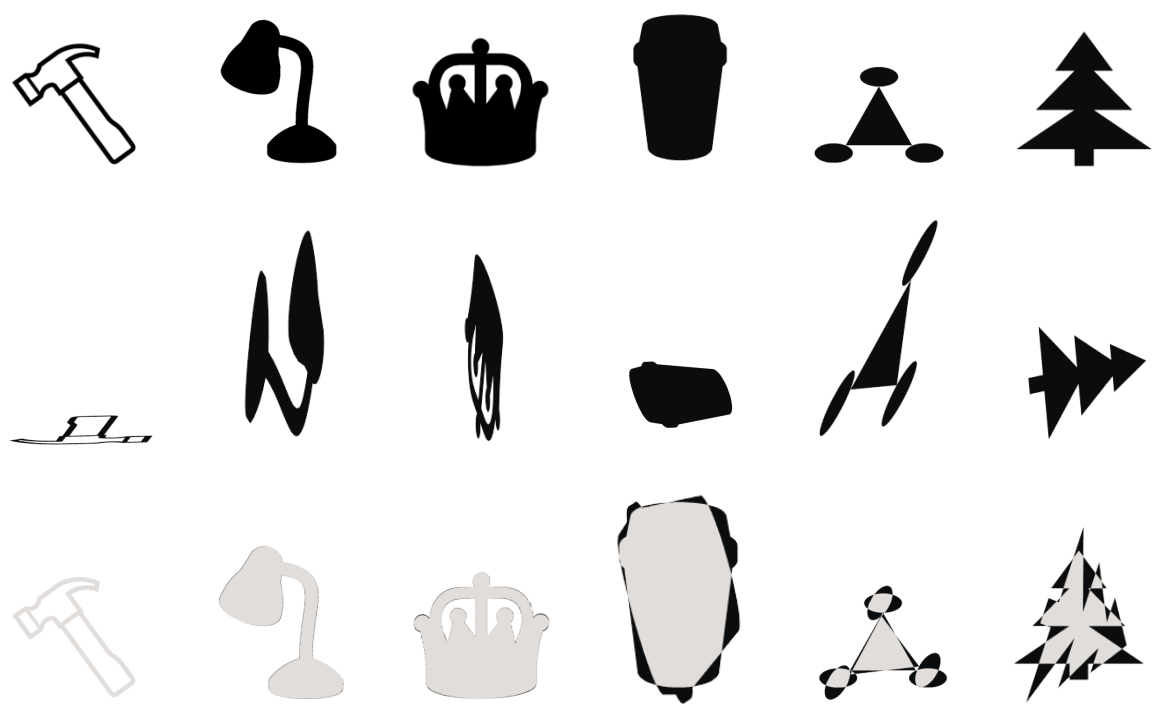

$\delta=0.17 \%$

$\delta=0.25 \%$

$\delta=1.1 \%$

$\delta=8.87 \%$

$\delta=23.79 \%$

$\delta=25.84 \%$

Fig. 1. Examples of templates (top row) and observations (middle row) images. In the third row, grey pixels show where the registered images matched each other and black pixels show the positions of registration errors.

respectively. We note that before computing the errors, the images were binarized by taking the $\alpha$-cut at $\alpha=0.5$ (in other words, by thresholding the membership function).

The medians of errors for both $\epsilon$ and $\delta$ are presented in Table 1 for different membership resolutions. For all membership resolutions, for around $5 \%$ of the images the system of equations provided no solution, i.e. the images were not registered. From the 56 images, there were only six whose transformed versions caused such problems. These can be seen in Fig. 2. Among the transformed versions, we found no rule to desribe when the problem occurs. Some of them caused problems for all different fuzzy membership resolutions, some of them occured for few resolutions only, randomly.

It is noticed that the experimental data confirmed the theoretical results, i.e. that the use of fuzzy shape representation enhances the registration, compared to the binary case. This effect can be interpreted as that the fuzzy representation "increases" the resolution of the object around its border. It also implies that registration based on fuzzy border representation may work for lower image resolutions, also where the binary approach becomes unstable.

Although based on solving a system of polynomial equations, the proposed method provides the result without any iterative optimization step or correspondence. Its time complexity is $\mathcal{O}(N)$, where $N$ is the number of the pixels of the image. Clearly, most of the time is used for parsing the foreground pixels. All 
Table 1. Registration results of 2000 images using different quantization levels of the fuzzy boundaries

\begin{tabular}{c|cccccccc} 
& \multicolumn{8}{|c}{ Fuzzy representation } \\
& 1-bit & 2-bit & 3-bit & 4-bit & 5-bit & 6-bit & 7-bit & 8-bit \\
\hline$\epsilon$ median (pixels) & 0.1681 & 0.080 & 0.0443 & 0.0305 & 0.0225 & 0.0186 & 0.0169 & 0.0147 \\
$\delta$ median (\%) & 0.1571 & 0.0720 & 0.0439 & 0.0292 & 0.0196 & 0.0151 & 0.0125 & 0.0116 \\
\hline Registered & 1905 & 1919 & 1934 & 1943 & 1933 & 1929 & 1925 & 1919 \\
Not registered & 95 & 80 & 66 & 57 & 67 & 71 & 75 & 81
\end{tabular}
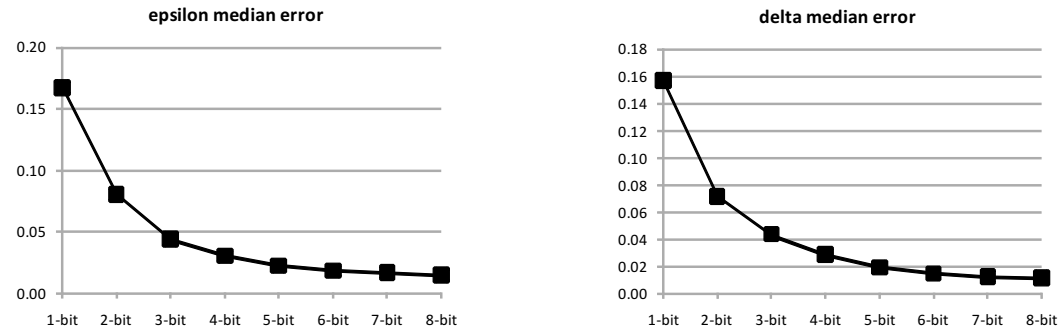

the summations can be computed in a single pass over the image. The algorithm has been implemented in Matlab 7.2 and ran on a laptop using Intel Core2 Duo processor at $2.4 \mathrm{GHz}$. The average runtime is a bit above half a second, including the computation of the discrete moments and the solution of the polynomial system. This allows real-time registration of $2 \mathrm{D}$ shapes.

\subsection{Experiments on Real X-Ray Images}

Hip replacement is a surgical procedure in which the hip joint is replaced by a prosthetic implant. In the short post-operative time, infection is a major concern. An inflammatory process may cause bone resorption and subsequent loosening or fracture, often requiring revision surgery. In current practice, clinicians assess loosening by inspecting a number of post-operative X-ray images of the patient's hip joint, taken over a period of time. Obviously, such an analysis requires the registration of X-ray images. Even visual inspection can benefit from registration as clinically significant prosthesis movement can be very small.
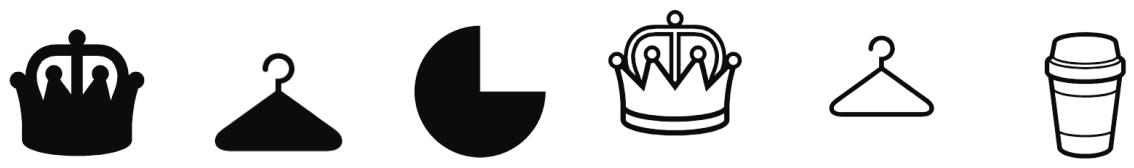

Fig. 2. Images where the polynomial system of equations provided no solutions in some cases. With increasing level of fuzzy discretization, the registration problem of the first three images vanished. The last three images provided problems permanently. 


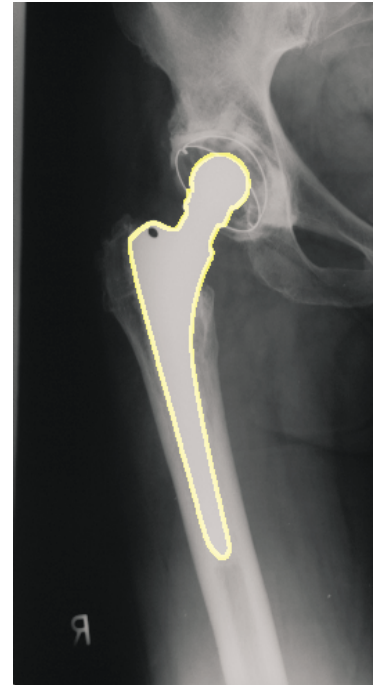

$\delta=2.17 \%$

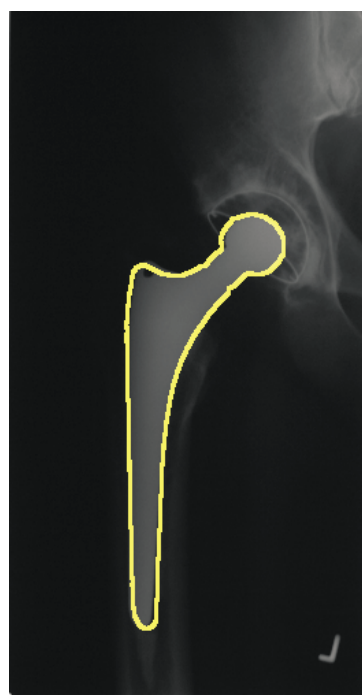

$\delta=4.81 \%$
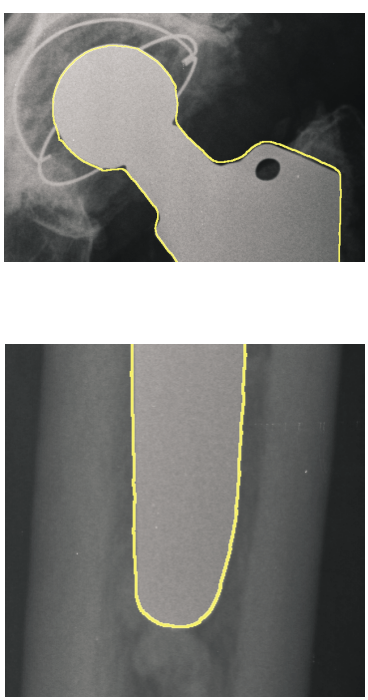

$\delta=1.2 \%$

Fig. 3. Real X-ray registration results. (a) and (b) show full X-ray observation images and the outlines of the registered template shapes. (c) shows a close up view of a third study around the top and bottom part of the implant.

There are two main challenges in registering hip X-ray images: One is the highly non-linear radiometric distortion 8] which makes any greylevel-based method unstable. Fortunately, the segmentation of the prosthetic implant is quite straightforward [9] so shape registration is a valid alternative here. Herein, we used the proposed fuzzy segmentation method to segment the implant. The second problem is that the true transformation is a projective one which depends also on the position of the implant in 3D space. Indeed, there is a rigid-body transformation in 3D space between the implants, which becomes a projective mapping between the X-ray images. Fortunately, the affine assumption is a good approximation here, as the X-ray images are taken in a well defined standard position of the patient's leg.

For for the diagnosis, the area around the implant (especially the bottom part of it) is the most important for the physician. It is where the registration must be the most precise. Fig. 3 shows some registration results. Since the best aligning transformation is not known, only the $\delta$ error measure can be evaluated. We also note, that in real applications the $\delta$ error value accumulates the registration error and the segmentation error. The preliminary results show that our approach using fuzzy segmentation and registration can be used in real applications.

\section{Conclusions}

In this paper we extended a binary affine shape registration method to take advantage of a discrete fuzzy representation. The tests confirmed expectations 
from the theoretical results of [4, on increased precision of registration if fuzzy shape representations are used. This improvement was demonstrated by a quantitative evaluation of 2000 images for different fuzzy membership discretization levels. We also presented a segmentation method based on Active Contour to generate fuzzy boundary representation of the objects. Finally, the results of a successful application of the method were shown for the registration of X-ray images of hip prosthetic implants taken during post-operative controls.

\section{References}

1. Zitová, B., Flusser, J.: Image registration methods: A survey. Image and Vision Computing 21(11), 977-1000 (2003)

2. Domokos, C., Kato, Z., Francos, J.M.: Parametric estimation of affine deformations of binary images. In: Proceedings of International Conference on Acoustics, Speech and Signal Processing, Las Vegas, Nevada, USA, pp. 889-892. IEEE, Los Alamitos (2008)

3. Hagege, R., Francos, J.M.: Linear estimation of sequences of multi-dimensional affine transformations. In: Proceedings of International Conference on Acoustics, Speech and Signal Processing, Toulouse, France, vol. 2, pp. 785-788. IEEE, Los Alamitos (2006)

4. Sladoje, N., Lindblad, J.: Estimation of moments of digitized objects with fuzzy borders. In: Roli, F., Vitulano, S. (eds.) ICIAP 2005. LNCS, vol. 3617, pp. 188-195. Springer, Heidelberg (2005)

5. Sladoje, N., Lindblad, J.: High-precision boundary length estimation by utilizing gray-level information. IEEE Transaction on Pattern Analysis and Machine Intelligence 31(2), 357-363 (2009)

6. Kass, M., Witkin, A., Terzopoulos, D.: Snakes: Active contour models. International Journal of Computer Vision 1(4), 321-331 (1988)

7. Boudier, T.: The snake plugin for ImageJ. software, http://www.snv.jussieu.fr/ wboudier/softs/snake.html

8. Florea, C., Vertan, C., Florea, L.: Logarithmic model-based dynamic range enhancement of hip X-ray images. In: Blanc-Talon, J., Philips, W., Popescu, D., Scheunders, P. (eds.) ACIVS 2007. LNCS, vol. 4678, pp. 587-596. Springer, Heidelberg (2007)

9. Oprea, A., Vertan, C.: A quantitative evaluation of the hip prosthesis segmentation quality in X-ray images. In: Proceedings of International Symposium on Signals, Circuits and Systems, Iasi, Romania, vol. 1, pp. 1-4. IEEE, Los Alamitos (2007) 